Revista de Comunicación y Salud, 2020, Vol. 10, nº 1, pp. 1-17

Editado por Cátedra de Comunicación y Salud

ISSN: 2173-1675

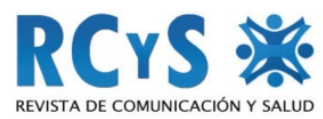

Enviado 21/04/2020

Aprobado 05/06/2020

\title{
REFERENTES CONCEPTUALES PARA EL ABORDAJE DE LA SALUD Y LA EDUCACIÓN ALIMENTARIA Y NUTRICIONAL EN LA ESCUELA
}

\section{Conceptual references for the approach to Health and Food and Nutrition Education at School}

\author{
Ernesto Elías De La Cruz Sánchez ${ }^{1}$ \\ Universidad Pedagógica Experimental Libertador. Venezuela \\ ernestodelacruz0203@yahoo.es
}

Financiación. Este trabajo es parte del proyecto: "La Educación Alimentaria y Nutricional como espacio de resonancia académico e investigativo en la UPEL" y contó con el apoyo del Fondo para el Fomento y Desarrollo de la Investigación, de la Universidad Pedagógica Experimental Libertador (UPEL). CNM632DL:2017-2019

\section{Resumen}

La problemática, hoy más que nunca, de educar en salud, nutrición y alimentación, constituye una prioridad para los países y así lo refieren organizaciones como la OMS, OPS y la FAO, entre otras. En tal sentido, en el presente trabajo analizamos en primer lugar, los referentes conceptuales de la salud como problema de la educación, su abordaje transdisciplinario, de enfoque salugénico, bajo un modelo social que tenga como centro de promoción y desarrollo a la escuela. En segundo lugar, destacamos la evolución del concepto de nutrición y su asociación con la alimentación desde la tridimensionalidad bilógico-social-ambiental que requieren, en el marco de enfoques sustentables-sostenibles, estar vinculados con la calidad de vida. Sin dejar de lado los efectos de la malnutrición, de las ECNT y la desnutrición fetal como causa epigenética potencial de dichas enfermedades. Por último, abordamos las diversas perspectivas que asume el hecho alimentario en nuestra sociedad, la cultura alimentaria, su aprendizaje social y cómo podemos entretejer estos escenarios en una verdadera pedagogía de la alimentación donde se articule la visión de la salud-alimentación como saber interdisciplinario propio y alcanzar una Educación Alimentaria y Nutricional como concepto transversal e integral que genere nuevos caminos de desarrollo, calidad de vida y bienestar desde la infancia.

\footnotetext{
${ }^{1}$ Autor para correspondencia: Ernesto Elías De La Cruz Sánchez. ernestodelacruz0203@yahoo.es. Profesor Titular en la Universidad Pedagógica Experimental Libertador. http://educacionan.blogspot.com/
} 
Referentes conceptuales para el abordaje de la salud y la educación alimentaria y nutricional en la escuela

Palabras Claves: salud, nutrición, alimentación, cultura alimentaria, malnutrición, pedagogía alimentaria, educación.

\begin{abstract}
Today, educating the public about health, nutrition, is a priority issue for countries, and so it is referred by organizations such as WHO, PAHO, and FAO, among others. With that in mind, in this paper nutritional and health concepts, like issues with education, the transdisciplinary and salutogenic approaches, are first analyzed, under a social model that has the school as a center of promotion and development. Secondly, are highlight the evolution of the concept of nutrition and its association with food from the biologicalsocial-environmental three-dimensionality that are required; in the framework of sustainable-sustainable approaches linked to quality of life. Without leave the effects of malnutrition as disease inflicted by fetal malnutrition and CNCDs as a potential epigenetic cause. Finally, are boarding diverse perspectives that the food fact assumes in our society, the food culture. Its social learning and how can interweave these scenarios in a true pedagogy of food where the vision of health-food is articulated as our interdisciplinary knowledge and Achieve Food and Nutrition Education as a transversal and integral concept that generates new ways of development, quality of life and wellbeing from childhood.
\end{abstract}

Keywords: health, nutrition, food, food culture, malnutrition, food pedagogy, education.

\title{
Cómo citar el artículo
}

De La Cruz Sánchez, E. E. (2020). Referentes conceptuales para el abordaje de la salud y la educación alimentaria y nutricional en la escuela. Revista de Comunicación y Salud, 10(1), 1-17. doi: http://doi.org/10.35669/rcys.2020.10(1).1-17

\section{LA SALUD COMO PROBLEMA DE LA EDUCACIÓN}

La Organización Mundial de la Salud (OMS) en su Carta Magna (1946), define la salud como "completo estado de bienestar físico, psíquico y social, y no solamente la ausencia de afecciones o enfermedades". Esto nos lleva a entender a la salud tanto como un hecho social como inmaterial. Hoy en día está aparentemente consensuada la definición de la OMS publicada en su formulación de objetivos de la Estrategia de Salud para Todos en el siglo XXI (OMS, 1997), donde se define a la salud como aquello a conseguir para que todos los habitantes puedan trabajar productivamente y participar activamente en la vida social de la comunidad donde viven.

Razón por la cual, estos referentes nos orientan a interpretar -reinterpretar- los conceptos de salud-enfermedad y la forma como se abordan, sobre todo desde la escuela, como categorías ideológicas, influenciadas por el contexto económico, político y social que los rodea, evolucionando y respondiendo a los cambios socio políticos actuales. Esta perspectiva, donde la salud es un componente de la calidad de vida, se nutre de la teoría de la complejidad y plantea abordar el análisis de la salud de las 
Referentes conceptuales para el abordaje de la salud y la educación alimentaria y nutricional en la escuela

poblaciones como espacio privilegiado, trabajando desde la teoría de sistemas complejos y adaptativos. Dicho enfoque, precisa lograr una articulación transdisciplinaria que innove, cree nuevos conocimientos y proyectos educativos que superen la yuxtaposición de saberes, habilidades y destrezas. Por su parte, en el enfoque sistémico de la salud, la concibe como un proceso social en el cual se produce y reproduce la vitalidad humana colectiva e individual, que conlleva dimensiones económicas, sociales, culturales, políticas y espirituales (González y Sosa, 2010).

En forma específica, se destaca la Estrategia mundial sobre régimen alimentario, actividad física y salud (OMS, 2004). La estrategia aborda dos de los principales factores de riesgo de las Enfermedades Crónicas No Transmisibles (ECNT): el régimen alimentario y la actividad física; parte del reconocimiento y preocupación por este problema y su impacto a nivel económico, social y cultural; a partir del cual propone: (a) reducir los factores de riesgo, (b) promover la conciencia y el conocimiento en la población, en torno al potencial positivo de una alimentación saludable y de la actividad física; (c) el establecimiento y el fortalecimiento de políticas y planes de acción en este sentido; y (d) el seguimiento, monitoreo, la investigación científica y la evaluación de las intervenciones para mejorar futuras acciones.

Por otro lado, cuando se quiere actuar sobre la salud reduciendo la incidencia, la duración, la intensidad o las consecuencias de enfermedades específicas que sufren los individuos, se requiere poseer la capacidad de evaluar el diseño, la implementación e impacto de las intervenciones que actúan sobre la salud, el funcionamiento biológico y psíquico de los seres humanos, sobre la base de un enfoque Salugénico -salutogénicoorientado a dotar a las personas de la capacidad de generar salud frente a la adversidad, teniendo como énfasis los orígenes (muchas veces socio-culturales) de la salud y el bienestar, en contraposición al enfoque Patogénico tradicional de la educación y promoción en salud orientado a la enfermedad (González y Sosa, 2010).

En apoyo a lo anterior, la OMS, emite en el año 2006, el mandato de utilizar las escuelas como un medio de fortalecimiento de la promoción de la salud, buscando mejorar así la calidad de vida de los estudiantes, las familias y los miembros de la comunidad. Promover el concepto de escuelas promotoras de salud, basado en un modelo social del bienestar, el cual debe hacer hincapié en la necesidad de reconocer a cada persona como centro de la organización y utilizando un enfoque integral y un ambiente de apoyo que influya positivamente en las percepciones y en la acciones de todos los involucrados: maestros, alumnos, padres y comunidad (OMS, 2006).

Razón por la cual, la Escuela se constituye en el lugar ideal y de mayor responsabilidad para el logro de una salud integral y la consolidación de hábitos que mejoren la calidad de vida, en razón de ser la escuela el escenario primordial de la educación para la salud y a su vez, se convierte en agente de la misma, al incidir de manera directa en el conocimiento y la comprensión de los factores condicionantes de la salud y el bienestar individual-colectivo, los cuales ubican con especial referencia a los vinculados con la Educación Alimentaria y Nutricional (EAN) desde la infancia. 
Referentes conceptuales para el abordaje de la salud y la educación alimentaria y nutricional en la escuela

\section{LA ALIMENTACIÓN Y LA NUTRICIÓN COMO CAMPO TRANSDISCIPLINARIO EN/PARA LA EDUCACIÓN}

De acuerdo con la concepción Iberoamericana, la nutrición es un amplio y complejo conjunto de fenómenos biológicos, psicoemocionales y socioculturales asociados con la obtención, asimilación y metabolismo de los nutrientes, por lo que su estudio es necesariamente interdisciplinario concurriendo en ella: la biología, la ecología, la historia, la política, la economía, la psicología, la antropología, la sociología y cualquier otra disciplina que aborde los factores que afecten a la nutrición (Bengoa, 2005).

Al describir la evolución del concepto de nutrición hasta llegar a la concepción de la tridimensionalidad del mismo. Se le consideraba como un proceso exclusivamente biológico, en la actualidad involucra además, aspectos sociales y ecológicos. Se destaca la convergencia entre el concepto de salud y nutrición, a partir de la concepción biopsicosocial del individuo, motivo por el que se enfatiza su importancia en el marco de la salud como herramienta preventiva y promocional que no solo incluye el aspecto informativo sino también la modificación de conductas con el objetivo de que el individuo adquiera estilos de vida saludables (Macías y col, 2009).

En razón de lo anterior, la Nutrición puede ser entendida como un estado de bienestar subjetivo e integral, producido por un suficiente aporte de nutrientes biológicos, afectivos, sociales, económicos y cognitivo-convivenciales, los cuales son expresión de procesos socioeconómicos, socioafectivos y culturales del ambiente donde se vive (De Tejada y col, 2013). Como consecuencia de lo descrito, una adecuada nutrición demanda un estilo de vida particular donde se integren dichas variables; con este fin se hace necesario que ocurra un proceso de concientización, desde la infancia, que conduzca a la toma de decisiones adecuadas y asertivas en relación con un proyecto de vida, orientado a la estructuración de entornos nutricionales y alimentarios saludables, aquí la escuela tiene un papel protagónico.

Desde otra perspectiva, en el escenario de la alimentación, las prácticas alimentarias no son solo hábitos, en el sentido de repetición mecánica de actos, iluminados por un positivismo ingenuo. Consecuentemente, no pueden interpretarse, como frecuentemente se ha hecho, como hábitos, más o menos inadecuados, sino que deben ser considerados como consecuencia también de razones culturales y se constituyen en sí mismo en patrimonio cultural de los pueblos (De La Cruz, 2017). Aparentemente, para la medicina y la nutrición, el ser humano se nutre sólo de glúcidos, lípidos y prótidos... pero lo cierto es que los alimentos, además de nutrir, significan y comunican. Los hábitos alimentarios son una parte integrada de la totalidad cultural. Somos lo que comemos y comemos lo que somos, (Montanari, 2004; Contreras y García, 2005).

Esta selección de alimentos que una determinada sociedad realiza de entre los diferentes recursos accesibles y comestibles se explica por razones técnicas y económicas. Pero también, se considera una cuestión de gusto o sabor y, muy a menudo, se explica por las creencias relativas a la bondad o maldad atribuidas a tal o cual alimento. También puede explicarse por el estatus de los alimentos en el seno de 
Referentes conceptuales para el abordaje de la salud y la educación alimentaria y nutricional en la escuela

los sistemas de organización y de funcionamiento de la naturaleza que las sociedades humanas han elaborado a lo largo de su historia (Harris, 2011).

En tal sentido, cuando se quiere emprender una campaña o programa de educación alimentaria y nutricional en el seno de una institución educativa, de un grupo étnico o cultural determinado, es muy importante conocer los aspectos simbólicos que los alimentos revisten, los hábitos alimentarios que han sido estandarizados en el curso de toda su historia y forman parte del comportamiento del individuo en el seno de una comunidad.

Si bien los cambios socioculturales acaecidos en la actual Modernidad, no es la evolución cultural, en ella misma, quien ha contribuido a perturbar los mecanismos reguladores, sino más bien la crisis cultural que atraviesan los países desarrollados y principalmente la desagregación o la desestructuración de los sistemas normativos y de los controles sociales que gobernaban tradicionalmente las prácticas y las representaciones alimentarias. Se perfila una crisis multidimensional del sistema alimentario y por ello de su salud -individual-colectiva-, con sus aspectos biológicos, ecológicos, psicológicos, sociológicos y esta crisis, se inscribe en una crisis de nuestra civilización (Fischler, 2002).

Más aún, la actual Modernidad alimentaria, ha defraudado la relación del hombre con su alimentación, ha desconectado los códigos alimentarios referidos a las categorías sociales y los signos, ritos, y prácticas han entrado en crisis extrema (Contreras y García, 2005). Situación que se expresa en altos niveles de obesidad, con un incremento, a nivel mundial, en las tasas de mortalidad asociadas a enfermedades no transmisibles (71\%), donde más del $85 \%$ de estas muertes "prematuras" ocurren en países de ingresos bajos y medianos (OMS, 2018), como contraparte la desnutrición y la alteración-destrucción del ambiente, son características cada vez más frecuentes de este fenómeno a nivel mundial.

Adicionalmente, existe una creciente evidencia que coloca a la nutrición como un factor ambiental que tiene una enorme incidencia en diversas enfermedades tanto crónicas como autoinmunes. El efecto se inicia a nivel epigenético durante el embarazo, debido a que la nutrición de la madre afecta el crecimiento del feto y puede impactar en su desarrollo durante toda la infancia. La hipótesis de la programación fetal propone que la desnutrición fetal es el resultado de la alimentación deficiente de la madre y a problemas de transferencia de nutrientes de la madre al feto. Se ha comprobado que hombres y mujeres que tienen mayor riesgo de padecer enfermedad cardio-metabólica, son aquellos que experimentaron privación temprana, tuvieron bajo peso al nacer o presentaron sobrepeso en la etapa infato-juventil. Así mismo, teorías más recientes respecto al impacto de la desnutrición en la inteligencia del niño, señalan que la misma altera el desarrollo intelectual por interferencia con la salud del individuo, con sus niveles de energía y con sus tasas de desarrollo motor; en suma, las condiciones socioeconómicas deficientes pueden exacerbar todos estos factores y permitir la perpetuación del daño ocurrido a edades tempranas de la vida. (Kennedy, 2016; Campoy, 2017; Spencer y col, 2017).

Revista de Comunicación y Salud, 2020, Vol. 10, n 1, pp. 1-17 
Referentes conceptuales para el abordaje de la salud y la educación alimentaria y nutricional en la escuela

Es momento para reflexionar y redefinir el papel protagónico de la escuela y el rol del docente -y sus formadores- desde la educación inicial, en la nueva conceptualización de la alimentación y su devenir como eje central de una sociedad más saludable. Podemos centrar estas ideas al destacar los referentes y fuentes de abordaje de la alimentación y nutrición, (ver figura 1).

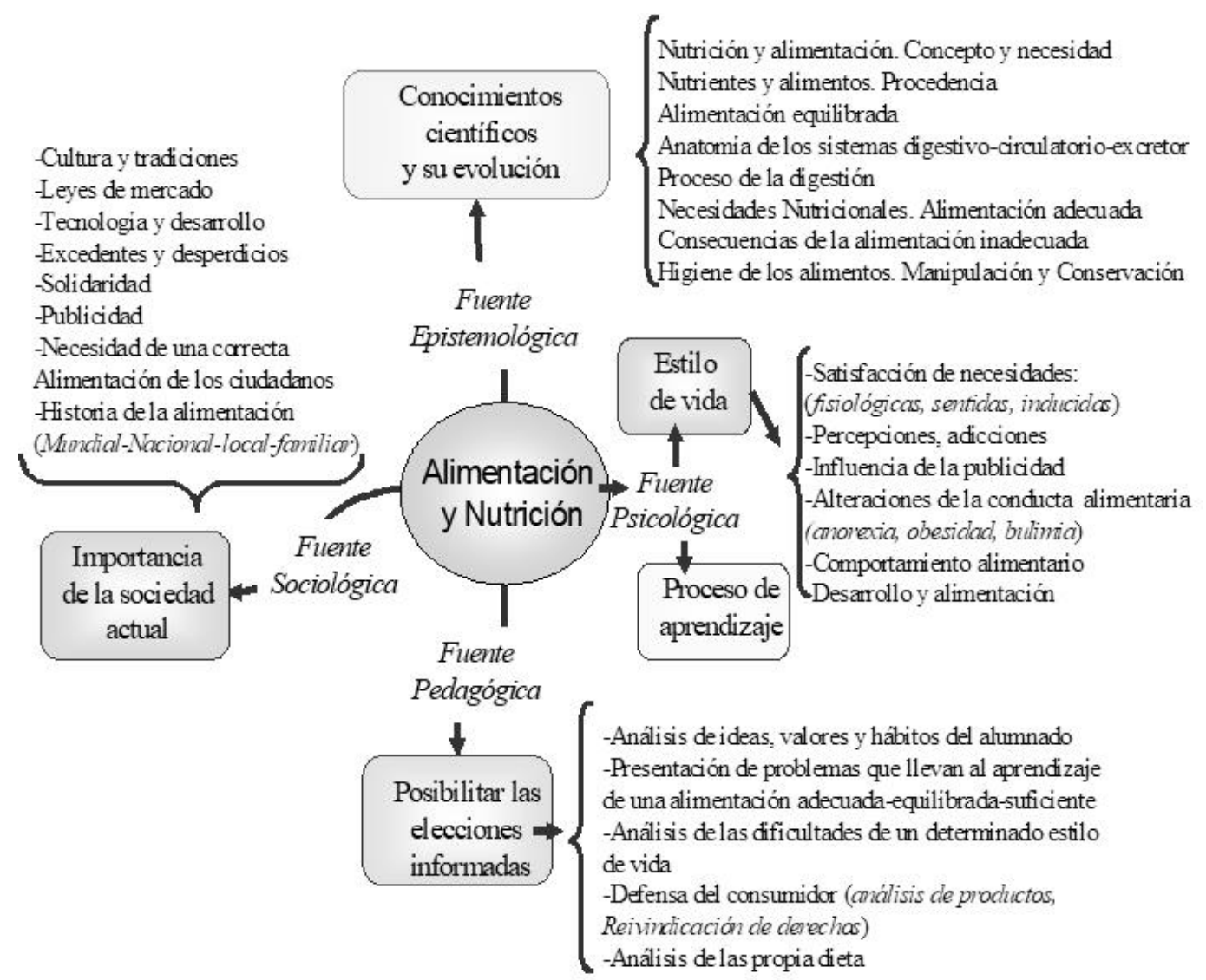

Figura 1. Esquema de las fuentes epistemológicas, psicológicas, sociológicas y pedagógicas de la alimentación y nutrición.

Fuente: Elaboración propia a partir de lo planteado por Gavidia y col (1993).

Para otros autores el énfasis de la EAN debe establecerse en su realización como derecho humano a una alimentación adecuada y de la garantía de una seguridad alimentaria y nutricional, entendida como la disponibilidad de alimentos suficientes, e inocuos. Esto conlleva a un campo de conocimiento y de reflexión continua y permanente, transdisciplinaria, intersectorial y multiprofesional que busque favorecer la práctica autónoma y voluntaria de hábitos alimentarios saludables. (Ministerio de Desarrollo Social-Brasil, 2012). Esta práctica debe hacer uso de abordajes y recursos educativos problematizadores, creativos y activos que promuevan el diálogo junto a individuos y grupos poblacionales, considerando todas las etapas del curso de la vida, del sistema alimentario, de las interacciones y de los significados del comportamiento alimentario. 
Referentes conceptuales para el abordaje de la salud y la educación alimentaria y nutricional en la escuela

Razón por la cual la Escuela se constituye en un escenario para desmontar los prejuicios asociados a creencias populares, falsamente tendidos por intereses variados y poco ajustados a criterios racionales. Precisamente, la escuela debe garantizar, por su condición de espacio de reconocimiento del saber público, que las personas se forman bajo parámetros de autonomía y emancipación pero en contextos sociales que requieren compromisos concretos y responsabilidades claramente delimitadas.

\section{CARACTERIZACIÓN DE LA EDUCACIÓN ALIMENTARIA Y NUTRICIONAL}

En líneas generales, la alimentación y la nutrición no son sinónimos. De acuerdo a los expertos la nutrición es el conjunto de procesos bioquímicos que se producen en todos los organismos de forma individual, involuntaria e inconsciente. La alimentación en cambio, es la forma -comportamientos- de llevar a cabo la nutrición y está influenciada por la cultura, la educación y el ambiente, entre otros. Más aún, la Nutrición hace referencia a los nutrientes que componen los alimentos y al conjunto de procesos involuntarios, como la digestión y la absorción de sus componentes o nutrientes, su metabolismo y la eliminación de los desechos celulares del organismo. Mientras que la alimentación se refiere al conjunto de actos voluntarios que abarca la elección del alimento, cómo lo preparamos, lo distribuimos y lo consumimos. El hecho de comer y el modo como lo hacemos tiene una gran relación con el ambiente en el que vivimos y determinan, en gran parte, los hábitos alimentarios y los estilos de vida de las personas, dichas ideas en conjunto se representan en la figura 2.

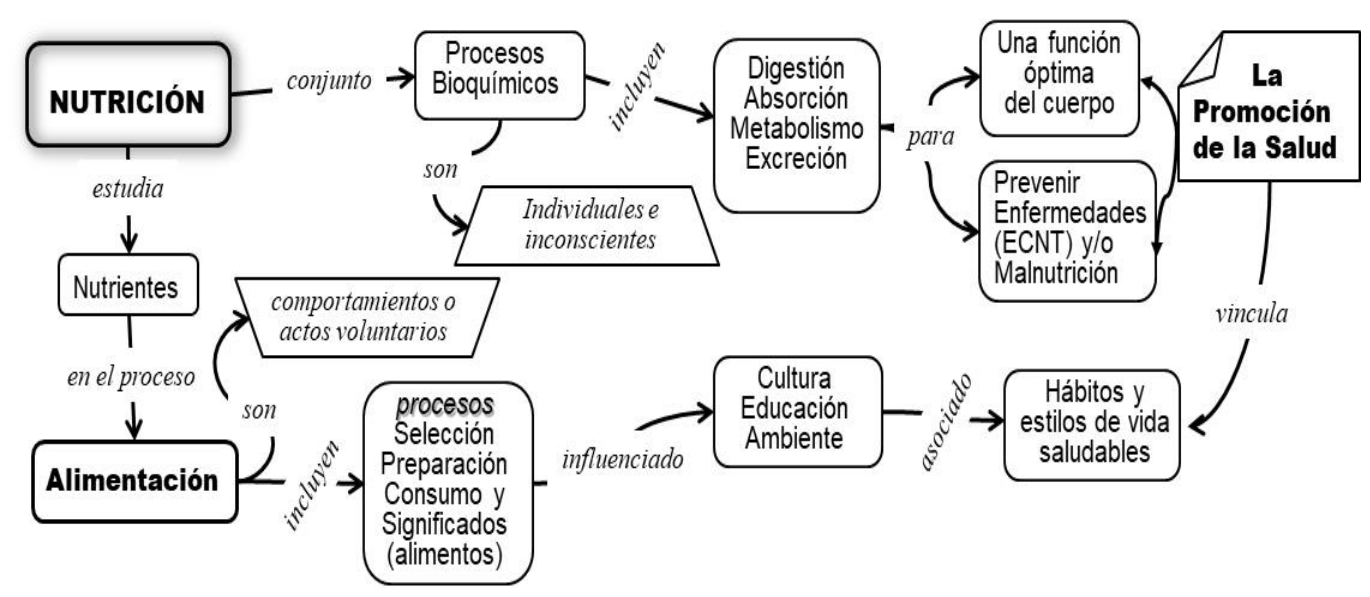

Figura 2. Caracterización tradicional de la nutrición y la alimentación en el campo de la educación.

Fuente. Elaboración propia.

Posteriormente, en el $17^{\circ}$ Congreso Internacional de Nutrición en Viena-Suiza, realizado en el año 2001, se reconceptualiza la idea de la nutrición, como el estudio de la totalidad de la relación entre las características funcionales del organismo (comportamiento metabólico) y su medio ambiente, dando énfasis al aporte calórico que 
proporcionan los alimentos, así como la importancia de una dieta saludable. Pero es en el año 2005, durante la Declaración de Giessen-Alemania-, que se propone la tridimensionalidad del concepto de la nueva ciencia de la nutrición, la cual es definida como el estudio de los alimentos y bebidas así como la constitución de otros alimentos y de las interacciones con los sistemas biológicos, sociales y ambientales más relevantes. De acuerdo con esta concepción la nutrición es un amplio y complejo conjunto de fenómenos biológicos, psicoemocionales y socioculturales asociados con la obtención, asimilación y metabolismos de los nutrientes, por lo que su estudio es necesariamente interdisciplinario (Beauman y col, 2005; Cannon y Leitzmann, 2005), (ver figura 3).

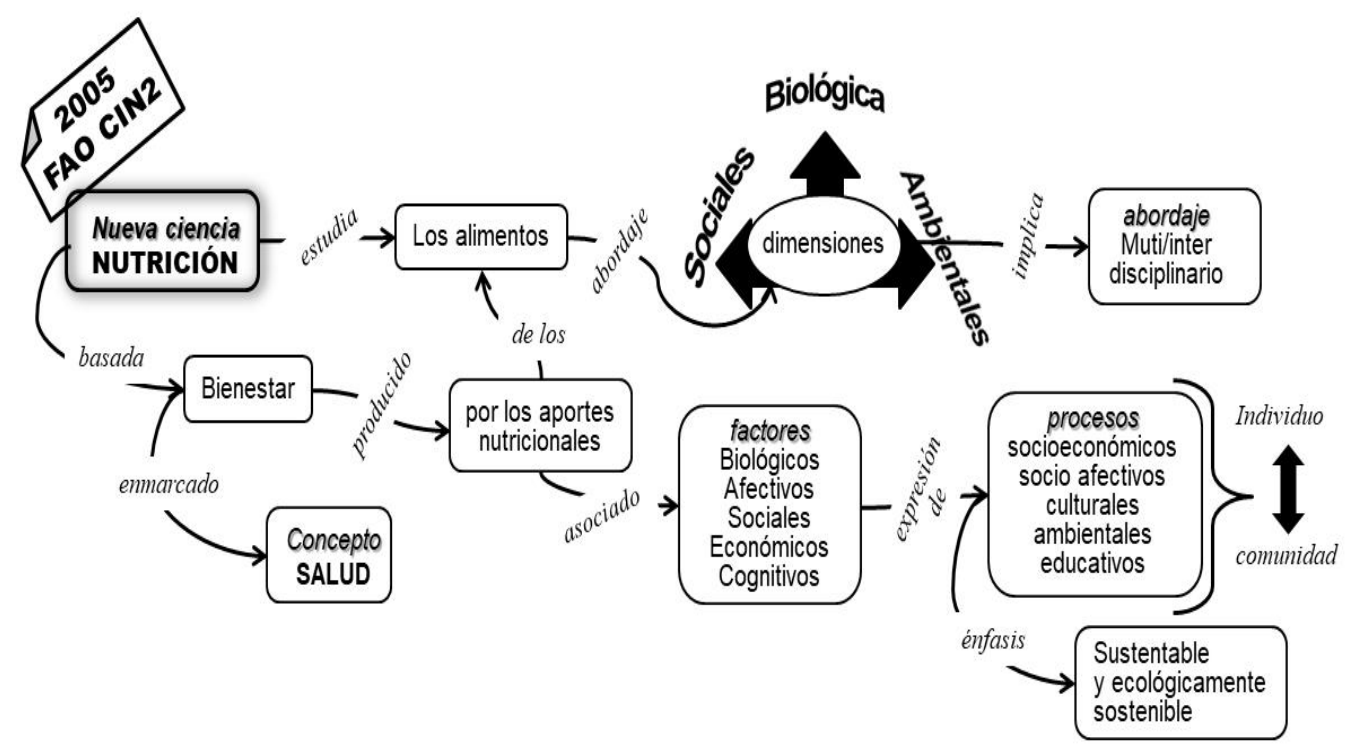

Figura 3. Nuevos referentes en la ciencia de la nutrición.

Fuente. Elaboración propia.

Estos referentes destacan la idea de tridimensionalidad en el abordaje de la nutrición, los cuales han sufrido considerables modificaciones durante estos años: (a) dimensión biológica: -eje central aún de la nutrición-, entendida como el conjunto de procesos mediante los cuales nuestro organismo, utiliza, transforma e incorpora a sus propios tejidos una serie de sustancias que recibe del mundo exterior y que han de cumplir tres fines básicos: aportar la energía necesaria para mantener la integridad y el funcionamiento de las estructuras corporales, construir y reparar estas estructuras y regular los procesos metabólicos; además de reducir el riesgo de algunas enfermedades a corto, mediano y largo plazo; (b) dimensión social: todos los países tienen una cultura alimentaria que influye en la elección de los alimentos y los hábitos de consumo. En este sentido intervienen determinantes sociales, culturales (religión y educación) y económicos que afectan a la nutrición de manera directa e indirecta, y que abarca desde las prácticas individuales a las poblacionales; y (c) dimensión ambiental: en la actualidad la disponibilidad de alimentos se ha visto afectada por las modificaciones en el medio ambiente, por ello la nueva nutrición sostenible implica que 
Referentes conceptuales para el abordaje de la salud y la educación alimentaria y nutricional en la escuela

sea ecológicamente sustentable. Esta perspectiva ecológica de la nutrición recomienda una alimentación sana, entendida como la manera más conveniente y sostenible de comer y beber, que conlleve a promover una dieta saludable, equitativa y sustentable (Macías y col, 2009).

Debemos de considerar, que hablar de nutrición nos remite indudablemente a pensar en la alimentación, entendida como la manera en que adquirimos los alimentos que nos proporcionan los nutrientes necesarios, que nos dan el aporte energético y los cuales tienen un fuerte componente social y cultural, por lo que es inevitable incluir en esta nueva perspectiva de la ciencia de la nutrición, un elemento importante como lo es: la promoción y la educación en materia de alimentación y nutrición, ya que en las tres dimensiones mencionadas (biológica-social-ambiental), se habla de reducir riesgos de algunas enfermedades mediante estrategias específicas como la sustentabilidad. Es por esto que una nueva estrategia a involucrarse es la educación, que no sólo va a tener como meta el informar si no también el de prevenir enfermedades crónicas generalmente provocadas por una inadecuada nutrición.

En el documento de la FAO, publicado en el año 2013, "retos y compromisos de la educación nutricional en el siglo XXI". Destaca que los problemas de deficiencias proteico-calóricas, de vitaminas y minerales, que conllevan a la malnutrición (por déficit o exceso), están relacionados con la dieta y las ECNT, que cada vez más coexisten en todo el mundo. En aquellos -sobre todo los niños- que no obtienen suficiente energía y nutrientes, desde su gestación, no pueden sostener vidas sanas y activas, tendrán un desarrollo físico y mental deficiente, así como una pérdida incalculable de su potencial humano, social y económico. Aquí el factor educativo reviste especial importancia.

Como apoyo de lo anterior se destacan los proyectos a nivel internacional, como los reportados por De Oliveira y Sousa (2008), "Niños Saludables", "Educación Diez" "Cocina Brazil" y "Alimentos Inteligentes"; Polanco y Pavón (2012), "el comedor escolar"; "gastronomía escolar" España y col (2014); Al-Ali y Arriaga (2016). En estos proyectos se destaca: (a) el uso del término "Educación Alimentaria y Nutricional" en lugar de "educación nutricional" ya que el centro del análisis y abordaje deben ser los alimentos y no sólo los nutrientes; (b) se debe promover el abordaje investigativo y educativo sobre alimentación y nutrición en diversos campos del conocimiento: antropología, historia, ambiente, psicología, economía, etc.; (e) dar prioridad a la Educación Alimentaria y Nutricional en las políticas públicas existentes y nuevas; y (f) promover proyectos políticos y pedagógicos que se centren en los alimentos, la educación alimentaria y nutricional, la formación docente y de especialistas en el área.

En las revisiones sobre la efectividad de 15 intervenciones en educación nutricional, en el mencionado documento, reflejaron que la educación en nutrición mejora los comportamientos en alimentación complementaria y el crecimiento infantil. Las intervenciones exitosas fueron culturalmente sensibles, accesibles e integradas con recursos y apoyo gubernamental. También se evidencia que la EAN es efectiva para modificar prácticas dietéticas que influyen en la prevención de enfermedades crónicas relacionadas con la alimentación. 
Referentes conceptuales para el abordaje de la salud y la educación alimentaria y nutricional en la escuela

Adicionalmente De la Cruz, (2019), en un análisis y revisión de las diversas definiciones del término de Educación Alimentaria y/o Nutricional, presentadas en la última década, por entes gubernamentales, declaración de profesionales, investigadores, ONG, publicaciones y libros de texto especializados. Llegó a la interpretación de que las definiciones en EN, van desde marcos de acción específicos (información, conocimientos) a descripciones complejas como disciplina, multifacética, interdisciplinaria, lo cual se refleja en la figura 4.

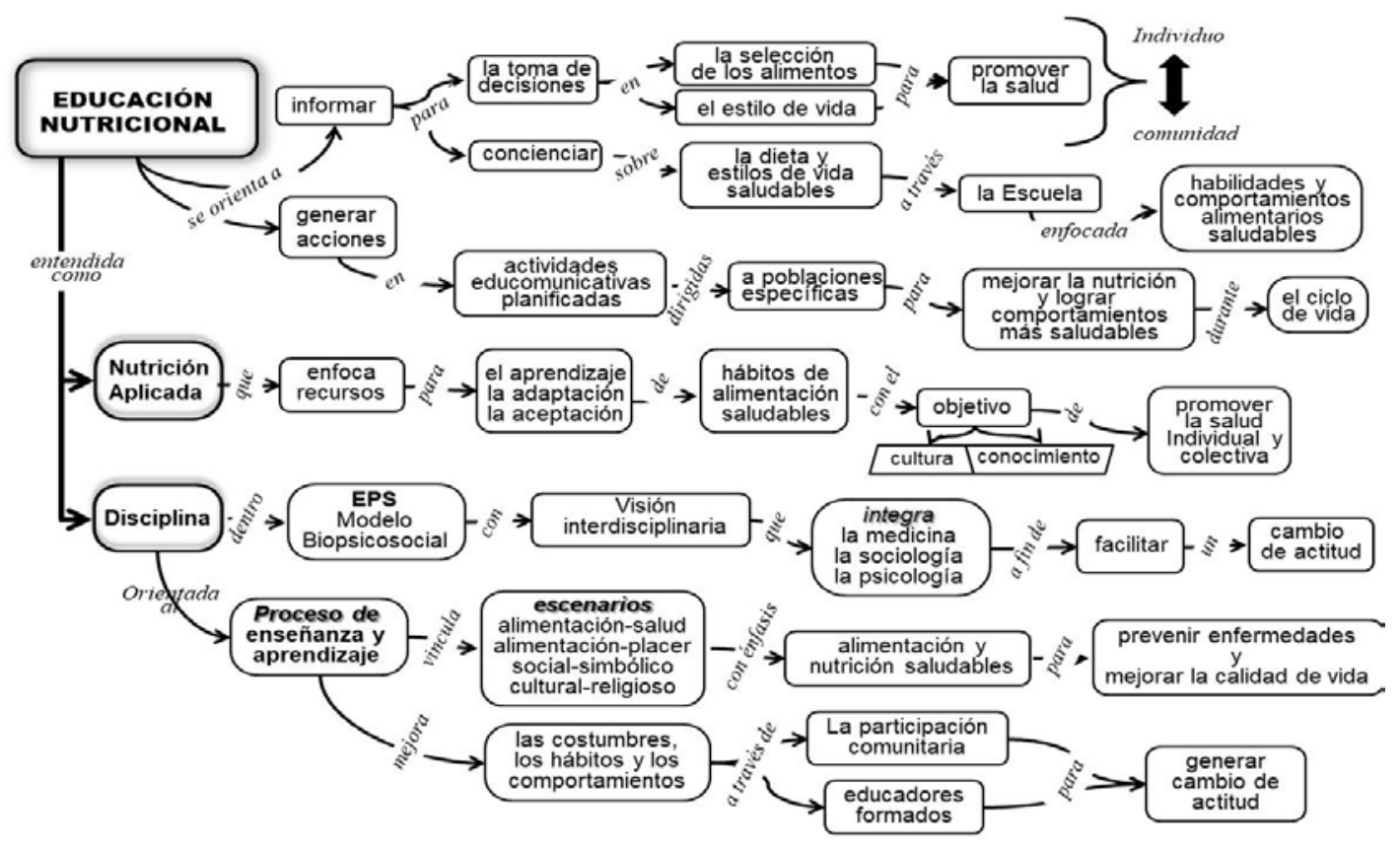

Figura 4. Elementos constituyentes de la Educación Nutricional. Fuente. Elaboración propia.

La mayoría de los autores, incluyen una dimensión de cambio de comportamiento o modificación voluntaria de las prácticas dietéticas. Sólo unas pocas referencias incluyen aspectos vinculadas a las políticas públicas. En su mayoría las acepciones implican que la EN proviene del exterior en lugar de constituirse en un aprendizaje autodirigido.

Para aclarar esta situación, se destacan las ideas de Alzate (2006), quien refiere la necesidad de reorientar el modo de pensar acerca de la EN, en este sentido se requiere realizar propuestas innovadoras, líneas de reflexión pedagógica que logren trascender la enseñanza temática y alcancen a tocar las intenciones, valores, principios vitales y sociales. Asumir la multicausalidad-multirespuesta-multisectorialidad y la participación social, sin olvidar el principio de autonomía del individuo y la responsabilidad colectiva en salud.

De sus consideraciones se extraen visiones comparativas entre los enfoque pedagógicos de la EN. La primera, de corte clásico, tuvo su auge en los años 70 fue elaborada por los teóricos de la salud y denominada difusionista-innovador, producto de 
Referentes conceptuales para el abordaje de la salud y la educación alimentaria y nutricional en la escuela

adaptaciones de los programas de extensión rural en Norteamérica, hacia países en desarrollo, basada en la denominada pedagogía tradicional, de la escuela nueva y tecnicista. El modelo clásico, de transmitir conocimientos puede asociarse con la pedagogía discursiva tradicional, donde la enseñanza está centrada en quien enseña como transmisor de contenido, o de modelo. Las categorías discursivas hablan de utilización adecuada de alimentos, importancia de la nutrición, transmisión de conocimientos, enseñar a alimentarse mejor, dar información, corregir, orientar, producir cambios en los conocimientos, actitudes y las prácticas en alimentación.

Posteriormente, surgen elementos con una visión más crítica, acciones transformadoras, que puedan aprovechar las experiencias populares, clases sociales, repensar la nutrición, cambios en las relaciones de producción-distribución de los alimentos. Planear, evaluar con los afectados y decidir con ellos. La EN como vehículo de discusión amplia, constituye una oportunidad de ofrecer un espacio para la reflexión de los problemas sociales en conjunto con la educación y la EN, ofrecer momentos para re-pensar el significado del binomio nutrición-alimentación, sus causas y consecuencias en ese grupo social, y como transformar los problemas más urgentes.

Por ello, la EAN debe orientarse a potenciar o modificar los hábitos alimentarios, involucrando a todos los miembros de la comunidad educativa; niños, padres, maestros y directivos. Educar sobre la necesidad e importancia de una buena alimentación implica: descubrir y erradicar creencias, mitos y conductas erróneas; promoviendo consciencia sobre las diversas funciones o roles que juega o debe jugar la alimentación en las diversas esferas de la vida, la salud, los aprendizajes, la producción, distribución y consumo de alimentos; fomentar conceptos, actitudes y conductas claras y fundamentales sobre la alimentación (De La Cruz, 2018), (ver figura 5).

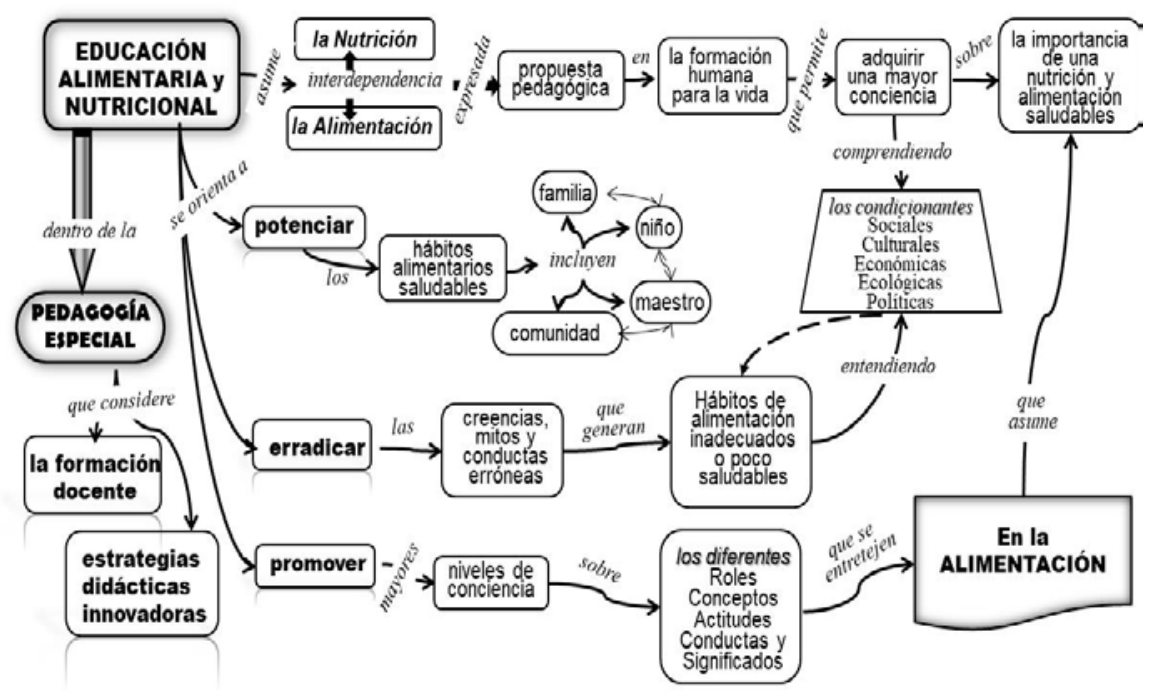

Figura 5. Elementos constituyentes y caracterizadores de la Educación Alimentaria y Nutricional.

Fuente. Elaboración propia. 
Referentes conceptuales para el abordaje de la salud y la educación alimentaria y nutricional en la escuela

Este componente debe ser abordado como un concepto transversal e integral, aprovechando todas las ramas del saber, nutriéndose de las cualidades sociales, culturales y antropológicas de los alimentos y a su vez le aporte, a estas dimensiones, nuevos enfoques. Que permita al niño un mayor conocimiento, actitud crítica y consolidación de sus hábitos; se forme con el ejemplo a través de mensajes saludables, con actividades continuas que logren el reforzamiento permanente y que lleven estos mensajes a la práctica.

Esta perspectiva educativa, debe tener como una de sus prioridades, la necesidad de consolidar hábitos adecuados de alimentación y nutrición durante toda la vida, a través de la sensibilización de los niños, de la familia y de la sociedad en torno a ella. Esto es factible a través de la estimulación del niño desde la infancia por medio del tacto, del olor, del gusto entre otros, ya que los alimentos constituyen una fuente de experiencias sensoriales, motrices y emocionales importantes, apoyándose en el fuerte componente cultural y simbólico que representan los alimentos para los niños-familia-comunidad, (FAO, 2013).

En este marco, en la Segunda Conferencia Internacional sobre Nutrición (Roma, 2018), se acordaron las siguientes recomendaciones: (a) implementar intervenciones de EAN, basadas en directrices dietéticas nacionales y políticas coherentes relacionadas con los alimentos y las dietas a través de mejores currículos escolares, servicios de protección social, intervenciones comunitarias e información de puntos de venta incluyendo el etiquetado; (b) realizar campañas de mercadeo social, programas de comunicación de cambios en el estilo de vida, promoción de la actividad física regular, consumo de alimentos ricos en micronutrientes como frutas y vegetales; (c) promoción del consumo de alimentos locales tradicionales y teniendo en cuenta los aspectos culturales; y (d) mejorar la nutrición infantil y materna, a través de prácticas adecuadas de cuidado, lactancia materna y alimentación complementaria.

Razón por la cual, la EAN es preciso considerarla en función de los factores que determinan el comportamiento alimentario del ser humano y que son la base de la enorme variabilidad de hábitos que presentan los individuos, entre los cuales están: la disponibilidad del alimento, factores sociales como la incorporación de la mujer al trabajo o la organización escolar, las modas, nuevos alimentos o abundancia de algunos de ellos, la publicidad de nuevos productos, las innovaciones en tecnología culinaria tales como el microondas, los factores religiosos y dentro de ellos podemos incorporar los modelos culturales, tradiciones y tabúes sobre los alimentos, y por último, el factor referido a las políticas públicas, nacionales-mundiales, en torno a la geoestrategia de la alimentación lo que incide en muchos casos en su costo económico y distribución. En todos estos escenarios los retos y compromisos que enfrenta la Educación, son hoy más que nunca impostergables.

\section{CONSIDERACIONES FINALES}

Cuando se quiere actuar sobre la salud reduciendo la incidencia, la duración, la intensidad o las consecuencias de las enfermedades específicas que sufren los 
Referentes conceptuales para el abordaje de la salud y la educación alimentaria y nutricional en la escuela

individuos, las intervenciones educativas son fundamentales y se trata, en este caso, de modificar el curso de los diferentes procesos biológicos y psíquicos afectados por la enfermedad, los cuales se asocian con los estilos de vida desde los enfoques Salugénicos propios del bienestar presente y futuro de nuestros niños.

Para ello tenemos que entender a la salud, no como un estado, sino como un proceso complejo y dialéctico; biológico y social; singular e interdependiente; alejado del equilibrio pero con cierto grado de estabilidad como consecuencia de mecanismos de adaptación y relaciones dinámicas, ecológicas, culturales, socioafectivos, políticas, económicas, vitales e históricas propias, en donde existe libertad de elección (autodeterminación del individuo).

Se debe abordar con mayor énfasis a la Educación Alimentaria y Nutricional en la escuela, como una propuesta estratégica en la formación humana para la vida. Como una línea de trabajo pedagógico que permita adquirir una mayor consciencia acerca de la importancia de la primera necesidad humana y aprendizaje social, lo cual nos debe inducir a revalorar la propia cultura alimentaria. Por ello, la EAN debe orientarse a potenciar o modificar los hábitos alimentarios, involucrando a todos los miembros de la comunidad educativa; niños, padres, maestros y directivos. Educar sobre la necesidad e importancia de una buena alimentación implica: descubrir y erradicar creencias, mitos y conductas erróneas; promoviendo consciencia sobre las diversas funciones o roles que juega o debe jugar la alimentación en las diversas esferas de la vida, la salud, los aprendizajes, la producción, distribución y consumo de alimentos; fomentar conceptos, actitudes y conductas claras y fundamentales sobre la alimentación, que permitan alcanzar a cada individuo su potencial como ser humano y donde la escuela debe cumplir un rol más comprometido y protagónico.

Por ello, organismos internacionales como la FAO-OMS (2018) han señalado que la EAN debe ser entendida como todas aquellas estrategias educativas diseñadas para facilitar la adopción voluntaria de conductas alimentarias y otros comportamientos relacionados con la alimentación y la nutrición propicios para generar mayores niveles de salud y el bienestar. Estas estrategias están enfocadas en el desarrollo de habilidades de los sujetos para tomar decisiones adecuadas en cuanto a su alimentación y en la promoción de un ambiente alimentario propicio. Dichas acciones se deben desarrollar o considerar los ámbitos individual, comunitario y político.

Lo cual requiere y requerirá una mayor reflexión acerca de cómo se aborda el acto pedagógico de la alimentación y la nutrición, profundizar en la formación crítica del docente en estos saberes fundamentales para la vida, valorar las historias alimentarias de los individuos, aportar soluciones creativas que permitan satisfacer sus necesidades fisiológicas, culturales, artísticas, sociales en materia de alimentación y nutrición, repensar en la salud-alimentación como un saber interdisciplinarios por excelencia para el logro del autodesarrollo del individuo, con el compromiso y responsabilidad de las instituciones formadores de docentes. 
Referentes conceptuales para el abordaje de la salud y la educación alimentaria y nutricional en la escuela

\section{REFERENCIAS}

Al-Ali, Nur y Arriaga, Andrés. (2016). Los elementos de efectividad de los programas de educación nutricional infantil. Revista Española de Nutrición Humana y Dietética. 20(1), 61-68. Disponible en: http://scielo.isciii.es/scielo.php?script=sci arttext\&pid=S217451452016000100008\&lng=es\&nrm=iso.http://dx.doi.org/10.14306/renhyd.20.1.181

Alzate, Teresita. (2006). Desde la Educación para la Salud: Hacia la Pedagogía de la educación alimentaria y nutricional. Perspectivas en Nutrición Humana. 16(2), 21-40. Disponible en: http://hdl.handle.net/10495/9846

Beauman C, Cannon G, Elmadfa I, et al. (2005). The principles, definition and dimensions of the new nutrition science. Public (Health Nutr. 8(6A), 695-698. doi:10.1079/phn2005820

Bengoa, José María, (2005) Algunos eslabones de su historia. Nutrición en América Latina. SLAN, 2005, pp13-34. Disponible en: www. sochinut.cl/pdf/HistNut SLAN.

Campoy, Cristina. (2017). Pregnancy Nutrition and Later Health Outcomes www. 112th Abbott Nutrition Research Conference .ANHI.org. Disponible en: https://static.abbottnutrition.com/cms-prod/anhi-2017.org/img/prenatal-nutrition-andcognition-pdf tcm1423-102253.pdf. Recuperado el 15 de agosto de 2019

Cannon, Geoffrey y Leitzmann, Claus. (2005). The new nutrition science project. Public Health Nutrition: 8(6A), 673-694. doi: https://doi.org/10.1079/PHN2005819

Contreras, Jesús y García, Mabel (2005). Alimentación y Cultura, perspectivas antropológicas. España: Ariel.

De La Cruz, Ernesto (2017). La Alimentación y Nutrición. Referentes de identidad social y patrimonio cultural. CLIC. 15(8), 129-138. Disponible en: https://convite.cenditel.gob.ve/revistaclic/index.php/revistaclic/anticle/view/888.

De La Cruz, Ernesto (2018). La Educación Alimentaria y Nutricional como Hecho Educativo. Revista Laurus. 1(1), 232-252. Disponible en: https://www.academia.edu/38454162/La Educaci\%C3\%B3n Alimentaria y Nutricion al como Hecho Educativo.

De La Cruz, Ernesto (2019). La Educación Alimentaria y Nutricional. Espacio de resonancia académico-investigativo. Trabajo de ascenso para optar a la categoría de profesor Titular, [inédito]. Universidad Pedagógica Experimental Libertador. Caracas, Venezuela.

De Tejada, Mirian; González, Humberto; De La Cruz, Ernesto; Rojas, Albino; LandaetaJiménez, Maritza; Pérez, Isabel y Delascio, Francisco. (2013). Educación Nutricional 
Referentes conceptuales para el abordaje de la salud y la educación alimentaria y nutricional en la escuela

para un desarrollo sostenible. Dirección de Publicaciones, Universidad Pedagógica Experimental Libertador. FEDEUPEL, Caracas, Venezuela.

De Oliveira, Sabrina y Sousa, Kathleen. (2008). Novas perspectivas em educação alimentar e nutricional. Psicol. USP. 19(4), 495-504. Sao Paulo. Disponible en: https://doi.org/10.1590/S0103-65642008000400008

España, Enrique; Ramos, Aurelio y Blanco Ángel. (2014). La competencia en alimentación. Un marco de referencia para la educación obligatoria. Enseñanza de las Ciencias. 32(2,3), 611-629. doi: http://dx.doi.org/10.5565/rev/ensciencias

FAO (2013). Prácticas en Información, Comunicación y Educación en Alimentación y Nutrición (ICEAN). Organización de las Naciones Unidas para la Alimentación y la Agricultura. Disponible en: http://www.fao.org/3/a-as503s.pdf

FAO-OMS (2018). Fortalecimiento de la acción nutricional. Una guía de recursos para países basada en las recomendaciones de políticas de la Segunda Conferencia Internacional sobre Nutrición (CIN2) Roma 2018. Disponible en: http://www.fao.org/publications/card/en/c/CA1505EN

Fischler, Claude. (2002). Gastro-nomia y gastro-anomia. Sabiduría del cuerpo y crisis biocultural de la alimentación contemporánea. En Contreras (2002) Alimentación y Cultura. Necesidades, gustos y costumbres. Cáp. V. p. 357-380. AlfaOmega Editores. México DF.

Gavidia, V., Rodes, M.J., y Carratalá, A., (1993). La Educación para la Salud: Una propuesta fundamentada desde el campo de la docencia. Enseñanza de las Ciencias. 11 (3), 289-296. Disponible en: file:///C:/Users/user/Downloads/21268-Texto\%20del\%20art\%C3\%ADculo-93547-110-20071030.pdf

González, María Cristina y Sosa, Gerónimo. (2010). Epistemología de la Salud Pública. Salusonline. 14-1 Abril. P.76. Disponible en: http://salus-online.fcs.uc.edu.ve/asp epis salud publica.pdf

Harris, Marvin. (2011). Bueno para comer. Editorial Alianza. Madrid, p. 252

Kennedy, David. (2016). B Vitamins and the Brain: Mechanisms, Dose and Efficacy- A Review. Nutrients. 8(2). 68-79. Disponible en:

https://www.ncbi.nlm.nih.gov/pmc/articles/PMC4772032/. https://doi.org/10.3390/nu8020068

Macías, Adriana; Quintero, María Luisa; Camacho, Esteban; y Sánchez, Juan. (2009). La tridimensionalidad del concepto de nutrición: su relación con la educación para la salud. Rev Chil Nutr. 36(4), 1129-1135. doi: https://dx.doi.org/10.4067/S0717$\underline{75182009000400010}$ 
Referentes conceptuales para el abordaje de la salud y la educación alimentaria y nutricional en la escuela

Ministerio del Desarrollo Social y Combate el Hambre (2012). Marco de referencia de Educación Alimentaria y Nutricional para las políticas públicas. - Brasilia, DF: MDS; Secretaria Nacional de Seguridad Alimentaria y Nutricional. Disponible en: http://www.fao.org/fileadmin/user upload/redicean/docs/marco EAN visualizacao es.pdf

Montanari, Massimo. (2004). La Comida como cultura. Ediciones TREA, S. L., España, pp. 982.

Organización Mundial de la Salud (1946). Carta Magna constitutiva. Disponible en: http://www.who.int/governance/eb/who constitution en.pdf

Organización Mundial para la Salud (1997). IV Conferencia Internacional sobra la Promoción de la Salud. Declaración de Yakarta, sobre la Salud Principal promoción en el siglo XXI. Disponible en:

https://www.who.int/healthpromotion/conferences/previous/jakarta/en/hpr jakarta dec laration sp.pdf

Organización Mundial de la Salud (2004). Estrategia mundial sobre régimen alimentario, actividad física y salud. Disponible en:

www.who.int/dietphysicalactivity/strategy/eb11344/strategy spanish web.pdf

Organización Mundial de la Salud OMS. (2006). Food and nutrition policy for schools. Copenhague. Disponible en:

http://www.schoolsforhalth.eu/...Who tod development nutrition program.pdf

OMS (2018). Enfermedades No Transmisibles. Centro de Prensa. Disponible en: https://www.who.int/es/news-room/fact-sheets/detail/noncommunicable-diseases

Polanco, Isabel y Pavón Pilar. (2012). Un reto actual: la prevención activa de la obesidad y el comedor escolar. En Nutrición en el ámbito escolar. Jesús Román Martínez Álvarez, (Editor). Cap 5, 59-67. España.

Spencer, Sarah; Korosi, Aniko; Leyé, Sophie; Shukitt-Hale, Bárbara y Barrientos, Ruth. (2017). Food for thought: how nutrition impacts cognition and emotion. npj Science of Food. 1(7). doi: https://doi.org/10.1038/s41538-017-0008-y

\section{AUTOR:}

Ernesto Elías De La Cruz Sánchez

Profesor en Biología y Ciencias Generales (UPEL); Postgrado en Evaluación Educacional; Magister en Ciencias de la Educación (UBA) y Doctor en Educación (UPEL); Posdoctorado en Educación Latinoamericana (RIEAC-UPEL). Jefe del Departamento de Ciencias Naturales y Matemática, IPMJM Siso Martínez (2003-2006).

Revista de Comunicación y Salud, 2020, Vol. 10, n 1, pp. 1-17 
Referentes conceptuales para el abordaje de la salud y la educación alimentaria y nutricional en la escuela

Coordinador de la Línea de Investigación en Educación Alimentaria y Nutricional (2010actualmente); autor-coautor de 4 libros, 6 capítulos de libros y 23 artículos publicados en el tema de la Educación Alimentaria y Nutricional (últimos 10 años). Miembro de la línea de Investigación "Estudio y Evaluación de la Creatividad" del Doctorado en Educación (UPEL). http://educacionan.blogspot.com/ ernestodelacruz0203@yahoo.es

Orcid ID: https://orcid.org/0000-0002-0197-331X

Academia.edu:

https://tecnoeducativa.academia.edu/ErnestoEl\%C3\%ADasDeLaCruzS\%C3\%A1nchez 